\title{
Ku complex suppresses recombination in the absence of MRX activity during budding yeast meiosis
}

\author{
Hyeseon Yun E Keunpil Kim ${ }^{*}$ \\ Department of Life Science, Chung-Ang University, Seoul 06974, Korea
}

During meiosis, programmed double-strand breaks (DSBs) are repaired via recombination pathways that are required for faithful chromosomal segregation and genetic diversity. In meiotic progression, the non-homologous end joining (NHE)) pathway is suppressed and instead meiotic recombination initiated by nucleolytic resection of DSB ends is the major pathway employed. This requires diverse recombinase proteins and regulatory factors involved in the formation of crossovers (COs) and non-crossovers (NCOs). In mitosis, spontaneous DSBs occurring at the $G 1$ phase are predominantly repaired via NHEJ, mediating the joining of DNA ends. The Ku complex binds to these DSB ends, inhibiting additional DSB resection and mediating end joining with Dnl4, Lif1, and Nej1, which join the Ku complex and DSB ends. Here, we report the role of the $\mathrm{Ku}$ complex in DSB repair using a physical analysis of recombination in Saccharomyces cerevisiae during meiosis. We found that the Ku complex is not essential for meiotic progression, DSB formation, joint molecule formation, or $\mathrm{CO} / \mathrm{NCO}$ formation during normal meiosis. Surprisingly, in the absence of the Ku complex and functional Mre11-Rad50-Xrs2 (MRX) complex, a large portion of meiotic DSBs was repaired via the recombination pathway to form COs and NCOs. Our data suggested that Ku complex prevents meiotic recombination in the elimination of MRX activity. [BMB Reports 2019; 52(10): 607-612]

\section{INTRODUCTION}

DNA double-strand breaks (DSBs) are the most toxic form of DNA lesions generated by various types of DNA damaging agents, such as free radicals, ultraviolet light, and ionizing radiation (1-4). To repair DSBs, cells induce tightly regulated

*Corresponding author. Tel: +82-2-820-5792; Fax: +82-2-820-5206; E-mail:kpkim@cau.ac.kr

https://doi.org/10.5483/BMBRep.2019.52.10.245

Received 21 October 2018, Revised 6 December 2018, Accepted 25 January 2019

Keywords: Double-strand breaks, Homologous recombination, $\mathrm{Ku}$ complex, Meiosis, Non-homologous end-joining
DNA repair programs such as homologous recombination and non-homologous end joining (NHEJ) that are highly conserved in all eukaryotic organisms. The choice of repair program between NHEJ and recombination depends on cell cycle phase and the process of DSB ends (5-7). For homologous recombination to proceed to repair spontaneous DNA damage and meiotic Spo11-catalyzed DSBs, many recombinase proteins and chromosome structural proteins play an important role. Homologous recombination utilizes the sister chromatid (or homologs in diploids) as a template for the repair of accidental DSBs during mitosis (8-11). Unlike mitosis, however, meiotic DSBs are repaired via the interhomolog recombination pathway to achieve genetic diversity for the next generation. In meiotic recombination, DSB ends predominantly utilize the homologous chromosome as a template for strand exchange to produce non-identical gametes by exchanging genetic information (12).

Meiotic recombination is initiated by programmed DSBs induced by the meiosis-specific topoisomerase II-like protein Spo11 (13). For recombination to progress, the highly conserved Mre11-Rad50-Xrs2 (MRX in yeast; Mre11-Rad50-Nbs2 in mammals) complex binds to DSB regions and controls end resection to remove Spo11 (14-16). DSB end resection during $\mathrm{S}$ and $\mathrm{G} 2$ phases of the cell cycle is achieved through cyclin-dependent kinase (CDK)-mediated phosphorylation of Sae2, an essential factor for activating the DNA endonuclease of the MRX complex, which is associated with bridging DNA ends (17-19). Exonucleases, such as Exo1 and Dna2-Sgs1, resect the 5'-ends of DNA strands to generate $3^{\prime}$ single-strand DNA (ssDNA) that is required for recombinase binding and homology searching (20). Replication protein A (RPA)-a heterotrimeric complex consisting of Rfa1, Rfa2, and Rfa3-binds to the ssDNA of DSB ends to inhibit secondary structures formed by ssDNA self-complementizing or to protect DSB ends from degradation (21). After displacement of RPA from ssDNA, Rad51, a RecA homolog, forms nucleofilaments that are also used for homology searching and homolog pairing during mitosis. However, in meiotic recombination, Rad51 functions as an auxiliary factor of Dmc1 for homolog bias (9).

NHEJ, a prominent DSB repair pathway of the mitotic cell cycle, mediates direct re-ligation of DSB ends from spontaneous DNA damage. NHEJ is initiated by a DNA end 
binding complex, the Ku70-Ku80 heterodimeric complex (Ku complex), which prevents $5^{\prime}$ strand resections of DSB ends $(17,22)$. Once the Ku complex binds to the DSB ends, it serves as a core site of NHEJ accessory factor recruitment to the DNA breaks. Inaccurate end-joining as a result of $\mathrm{Ku}$ complex-deficiency causes chromosomal breaks and aneuploidy (23). In budding yeast, DNA end processing involved in the NHEJ pathway is mediated by diverse factors including Dnl4 (ATP-dependent ligase; DNA ligase IV in vertebrates), Lif1 (XRCC4 in vertebrates), Nej1 (XLF in vertebrates), and Pol4 (Pol $\mu$ and Pol $\lambda$ in vertebrates) (24-26). Haber and colleagues reported that diploid yeast cells suppressed expression of Nej1 and Lif1, but ectopic expression of Nej1 restored NHEJ pathway to diploid cells (27). The DNA end bridge complex is targeted by a DNA ligase complex that mediates end-joining of DSB ends and inhibits DSB end resection, which is processed by nuclease-helicase enzymes (24-26). Finally, Pol4 and Lig4 are required for filling in the DNA gaps (25). In mammalian cells, DSB repair via homologous recombination utilizes the sister chromatid as a template because it is nearby during the S/G2 phase, while NHEJ is the major DSB repair process that occurs in all cell cycle phases. The Ku complex binds to a DNA end to form the Ku:DNA complex that serves as a platform where a ligase complex including XLF, XRCC4, and DNA ligase IV can dock to rejoin the ends $(25,26)$.

Here, we investigated the role of the Ku complex and the relationship between the $\mathrm{Ku}$ and MRX complexes. Experimental studies of NHEJ-mediated meiotic DSB repair are challenging because recombination is the major DSB repair pathway in meiosis. To this end, we present a NHEJindependent role of the Ku complex in meiotic recombination of Saccharomyces cerevisiae through physical analysis of recombination.

\section{RESULTS}

\section{The Ku complex is not essential for division and spore viability in normal meiosis}

The Ku complex rapidly localizes to DSB sites and is involved in protecting DNA ends from nuclease-helicase processing as well as recruiting NHEJ proteins (25). To provide insights into the role of the $\mathrm{Ku}$ complex during meiosis, we observed meiotic division and spore formation in wild-type (WT) and ku704 mutant cells (Fig. 1A and 1B). Meiosis was induced in cells incubated in sporulation medium (SPM) that were then harvested from the culture at different time points $(0,2.5,3.5$, 4, 5, 6, 7, 8, 10, and 24 h). In WT cells, meiotic division began after $5 \mathrm{~h}$ in sporulation media and rapidly progressed with $50 \%$ of cells having underwent division after approximately $6 \mathrm{~h}$. In ku704 cells, normal nuclear division occurred with a slight delay of about 20 min compared with that of WT (Fig. 1A). Moreover, DAPI staining indicated that both WT and ku70 4 cells exhibited normal nuclei separation after $24 \mathrm{~h} ; 91.4 \%$ and $4.2 \%$ of $k u 70 \Delta$ cells produced four and
A

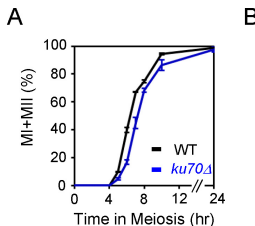

B

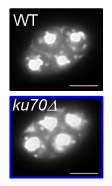

$C$
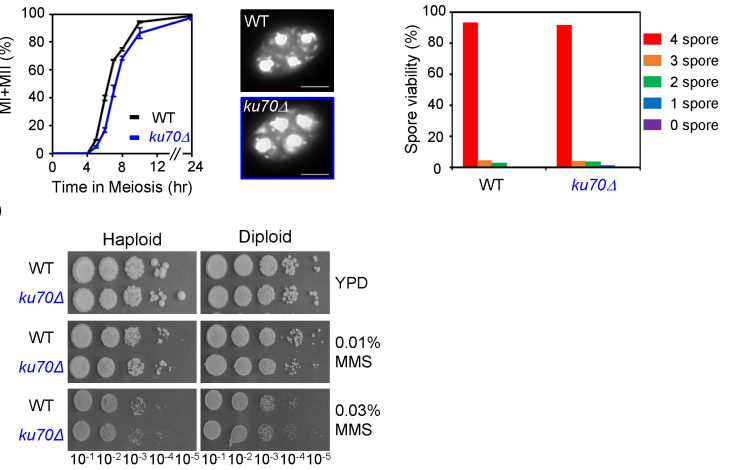

Fig. 1. Ku70 is not essential for meiotic progression. (A) Meiotic progression of WT and ku704 cells. Meiosis was induced in synchronized yeast in SPM and cell divisions were counted at the indicated time points. The error bar represents the standard deviation (SD; $\mathrm{n}=3$ ). (B) Representative images of DAPI-stained nuclei of WT and ku70 4 strains cultured in SPM for $24 \mathrm{~h}$. Scale bar $=2.5 \mu \mathrm{m}$. (C) Analysis of spore viability in WT and ku70 strains ( $\mathrm{n}>100)$. (D) MMS sensitivity test. Cells were serially diluted and spotted onto YPD plates and YPD plates containing $0.01 \%$ and $0.03 \%$ MMS.

three spores, respectively, compared with the $92 \%$ and $3 \%$ of WT, respectively (Fig. 1B and 1C). Thus, ku70 $\Delta$ cells underwent meiosis normally and formed viable spores as did the WT, confirming that NHEJ is not an essential pathway for repairing Spo11-induced DSBs. To understand the role of the Ku complex in mitotic DNA damage repair, we employed the methyl methane sulfonate (MMS) sensitivity test in the absence of the Ku complex (Fig. 1C and 1D). ku70 $\Delta$ cells grew at similar levels as the WT in YPD media containing $0.01 \%$ and $0.03 \%$ MMS (Fig. 1D). Thus, DNA damage of vegetative cells is not lethal for ku70 4 mutants, indicating that NHEJ is not an essential pathway in MMS-induced DSB repair.

\section{Physical analysis of meiotic recombination}

To determine the molecular pathway involved in meiotic recombination, we monitored recombination intermediates and final outcomes (crossovers [COs] and non-crossovers [NCOs]) using the HIS4LEU2 assay system for chromosome III (Fig. 2). In the HIS4LEU2 assay system, COs and NCOs can be detected after digesting genomic DNA with Xhol and NgoMIV enzymes. After synchronizing yeast cells at the G1 phase in pre-sporulation medium (SPS), the cells were transferred to sporulation medium to initiate meiosis. Cells were then treated with psoralen after harvesting to produce interstrand-crosslink DNA, which stabilizes single-end invasions (SEIs) and double-Holliday junctions (dHJs; 8-10, 28, 29). Meiotic DNA samples were digested with Xhol and then DNA fragments were analyzed by DNA gel electrophoresis and Southern blotting using Probe A (Fig. 2A and 2B). DNA species of interest, DSBs and COs, were quantified using a phospho- 
A

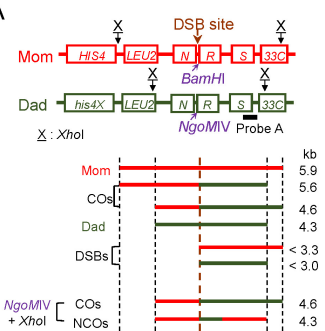

D
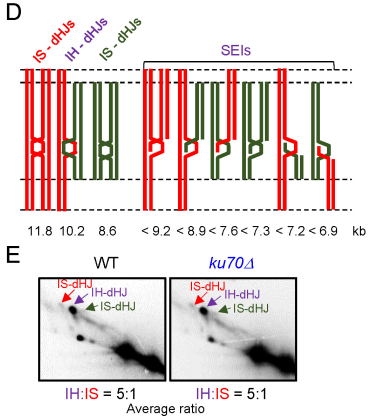

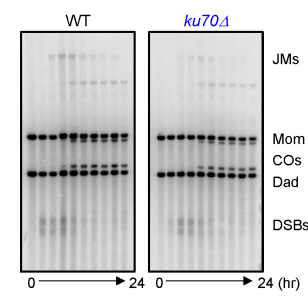

C

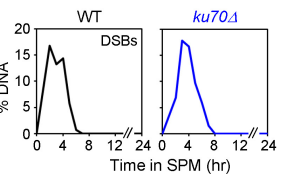

F

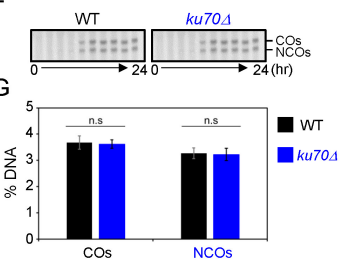

Fig. 2. Normal progression of meiotic DSB repair and formation of COs and NCOs in the absence of Ku70. (A) Physical map of the recombination assay for chromosome III. The HIS4LEU2 hotspot schematic includes restriction enzyme polymorphisms and the Southern blot probe (probe A). (B) 1D gel electrophoresis of WT and ku70 4 strains. Cells were harvested at different time points $(0,2.5,3.5,4,5,6,7,8,10$, and 24 h). (C) Quantitative analysis of DSBs in WT and ku704 cells. (D) Structure of the 2D gel analysis of the HIS4LEU2 locus. (E) 2D gel electrophoresis of WT cells. The average ratio of $\mathrm{IH}: \mathrm{IS}-\mathrm{dHJ}$ was 5:1 for both WT and ku704 cells. (F) Gel analysis of COs and NCOs. (G) Quantification of $\mathrm{IH}-\mathrm{CO}$ and $\mathrm{IH}-\mathrm{NCO}$ in WT and ku704 cells. Error bars represent SD $(n=3)$ and significance was determined by a Student $t$ test. Maternal species, paternal species, DSBs, double-strand breaks; IH-COs, interhomolog crossovers; IH-NCOs, interhomolog non-crossovers. SEI, single end invasion; $\mathrm{IH}-\mathrm{dHJ}$, interhomolog-double Holliday junction; IS-dHJ, intersister-double Holliday junction.

image analyzer. Parental DNA species were detected at $5.9 \mathrm{~kb}$ for maternal chromosomes and $4.3 \mathrm{~kb}$ for paternal chromosomes (Fig. 2A and 2B). DSB signals appeared at 3.0 $\mathrm{kb}$ and $3.3 \mathrm{~kb}$ in one-dimensional (1D) gel electrophoresis. Native/native two-dimensional (2D) gel electrophoresis was performed to detect joint molecules (JMs; SEI and dHJ; Fig. 2D and $2 \mathrm{E})$. COs and NCOs were distinguished in 1D gels at 4.6 $\mathrm{kb}$ and $4.3 \mathrm{~kb}$, respectively (Fig. $2 \mathrm{~A}$ and $2 \mathrm{~F}$ ).

\section{Repair of meiotic double-strand breaks progressed normally in $k u 70 \Delta$ cells}

In WT cells, DSBs were initiated after $2.5 \mathrm{~h}$ and peaked at $4 \mathrm{~h}$ with approximately $16.7 \%$ hybridizing DNA species that then disappeared after $6 \mathrm{~h}$. DSB levels and turnover were similar between WT and ku70 $\Delta$ cells (Fig. 2B and 2C). Similar data for another set of physical analysis of independent time course

A

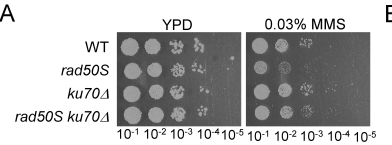

B

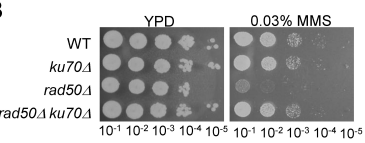

C

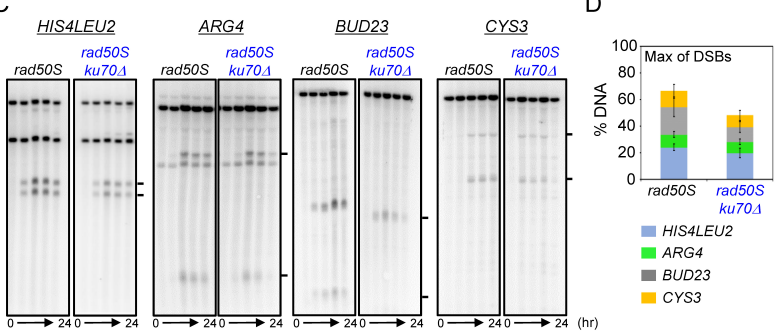

Fig. 3. The absence of Ku70 reduces DSB levels in a rad50S background. (A) MMS sensitivity test of rad50S and rad50S ku704

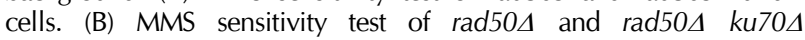
cells. (C) 1D gel electrophoresis of rad50S and rad50S ku70 4 of the HIS4LEU2, ARG4, BUD23, and CYS3 loci. (D) Maximum level of DSBs at each hotspot locus. Error bars represent SD $(n=3)$.

experiments are presented in Supplemental Fig. 1. The maximum level of COs and NCOs in WT cells was $3.8 \%$ and $3.1 \%$, respectively. The levels and turnover of COs and NCOs in ku704 cells were similar to those of WT cells, consistent with our meiotic division findings (Fig. 1 and Fig. 2G). To examine whether the $\mathrm{Ku}$ complex affects homolog bias, we performed 2D gel electrophoresis (Fig. 2E). Several types of JMs were detected using 2D gel analysis including intersister SEIs (IS-SEIs), IH-SEIs, IH-dHJs, and IS-dHJs. Consistent with our previous results for WT cells, $\mathrm{IH}-\mathrm{dHJ}$ levels were higher than IS-dHJs at a ratio of 5:1 (Fig. 2E). Moreover, the ratio of $\mathrm{IH}-\mathrm{dH} \mathrm{Js}$ and IS-dHJs in ku704 cells was also 5:1. Additionally, IH-SEls occurred at high levels in both WT and ku70 $\Delta$ cells. Thus, the results indicate that the Ku complex is not required for the formation of JMs (DSB-to-JM transition) and establishment of homolog bias.

\section{DSBs levels are reduced at the HIS4LEU2, ARG4, BUD23,} and CYS3 loci in rad50S ku70 4 cells

In rad50S mutant cells, the MRX complex is inactivated and thus DSBs accumulate instead of forming CO and NCO recombinants (30). Thus, the total number of DSBs can be measured from the rad50S allele, which is blocked at the DSB-to-JM transition. Surprisingly, the rad50S cells exhibited strong MMS sensitivity, but the ku70 4 mutation partially suppressed DNA damage of rad50S cells (Fig. 3A). Similar results were obtained when rad50 $\Delta$ and rad50 $\Delta$ ku70 $\Delta$ cells were examined in the same experiments (Fig. 3B). These results indicate that damaged DNA is possibly repaired in the deficiency of $\mathrm{Ku}$ complex and DSB resection. To investigate whether the Ku complex is required for DSB formation, we used 1D gel electrophoresis for rad50S DSB analysis at the HIS4LEU2, ARG4, BUD23, and CYS3 loci (Fig 3C and 3D). 
Notably, total levels of DSBs in rad50S ku704 cells were lower than those of WT cells at all loci. Furthermore, a significant subset of DSBs in rad50S ku704 cells were repaired to form COs at the HIS4LEU2 hotspot, which can distinguish between $\mathrm{IH}-\mathrm{CO}$ and $\mathrm{IH}-\mathrm{NCO}$ (Fig. 4A and 4B). Thus, a portion of DSBs in rad50S ku70 4 cells progressed to form COs at a later time point, indicating that cells repaired DSBs via the recombination pathway.

\section{Ku70 is involved in DSB repair during arrest of the DSB end} resection process

At the HIS4LEU2 locus in rad50S ku704 cells, a subset of
A

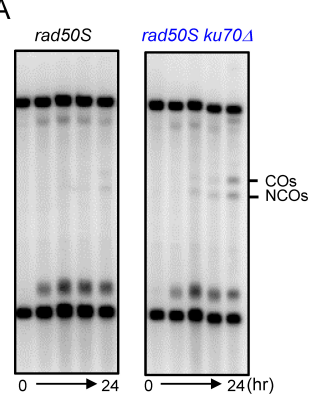

D
B

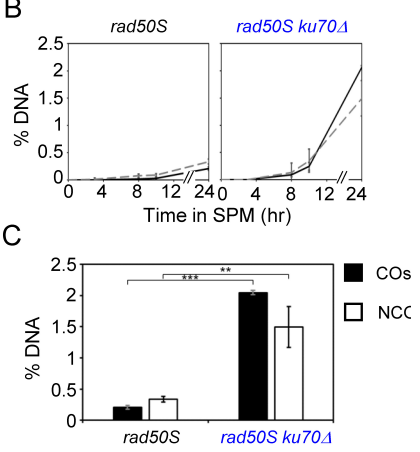

Fig. 4. Absence of Ku70 and a functional MRX complex promotes $\mathrm{CO}$ and NCO formation. (A) Representative images of $\mathrm{CO}$ and NCO gels in rad50S and rad50S ku704. (B) Quantitative analysis of COs and NCOs in rad50S and rad50S ku704 cells. Error bars represent SD $(\mathrm{n}=3)$. (C) Maximum level of COs and NCOs in rad50S and rad50S ku70 4 cells. Error bars represent SD $(\mathrm{n}=3)$ and significance was determined by a Student $\mathrm{t}$ test. $* * \mathrm{P}$ $<0.01,{ }^{*} * \mathrm{P}<0.001$. (D) Proposed model for the roles of the MRX complex and NHEJ pathway in meiotic recombination. DSBs are catalyzed by Spo11 and the MRX complex plays a role in DNA resection and Spo11-oligonucleotide release (34). Exo1 and the Dna2-STR complex promote additional DSB end resection to create long ssDNA overhangs. In the absence of NHEJ and a functional MRX complex, $\sim 50 \%$ of DSBs progressed to recombination to form COs and NCOs (this study). STR, Sgs1-Top3-Rmi1; SDSA, synthesis-dependent strand annealing.
DSBs progressed to COs at a much later time point (Fig. 4A). This finding suggests that the COs detected in rad50S ku704 cells may result from meiotic recombination, implying that nucleolytic resection of DSB ends occurred in the absence of the $\mathrm{Ku}$ complex and a functional MRX complex. We further investigated the formation of COs and NCOs in rad50S ku704 mutant cells. Notably, COs and NCOs were detected in rad50S ku70 4 cells (Fig. 4B and 4C). In rad50S ku704, NCOs appeared after approximately $8 \mathrm{~h}$ and COs appeared after 10 $\mathrm{h}$, indicating that NCOs formed earlier than COs. Interestingly, the maximum levels of COs were attained by $24 \mathrm{~h}$. Therefore, our findings indicate that DSB repair occurred to form CO and NCO through meiotic recombination in rad50S ku70 4 mutant cells.

\section{DISCUSSION}

DSBs can arise from diverse reactive metabolites, ionizing radiation, or stalling of DNA replication during cell cycle. Inappropriate repair of DSBs leads to cell death, senescence, or cancer. Two distinct DNA repair pathways, NHEJ and homologous recombination, eliminate DSBs depending on the cell cycle phase or the nature of DSB end process. During meiosis, cells induce programmed DSBs that are generated by Spo11 and accessory factors. The post-DSB role of Exo1 and the MRX complex is essential for promoting recombination. It has been reported that the MRX complex, in coordination with Sae2, mediates ssDNA nick formation and exhibits $3^{\prime}$ to $5^{\prime}$ exonuclease activity that resects the ssDNA towards Spo11-binding regions. Additionally, Exo1 and the Dna2-Sgs1 complex promote formation of long stretches of ssDNA that can be used for homology searching on homologous chromosomes during meiosis. The long single-stranded overhangs of DSBs are bound by the homology search and strand exchange proteins Rad51, Dmc1, and accessory factors including Rad52, Rad54, Rad54, Rad57, the PCSS complex, Hed1, Rdh54/Tid1, Hop-Mnd1, and Mei5-Sae3 (21). The MRX/N complex has been implicated in NHEJ-mediated DSB repair during mitosis in budding yeast. However, Ku complexmediated NHEJ is dispensable in meiotic recombination of budding yeast (Fig. 4D), whereas it is essential for the successful maintenance of genomic integrity in mammalian cells (26). The absence of NHEJ and a functional MRX complex in Caenorhabditis elegans channeled meiotic DSB repair to the exonuclease-dependent recombination pathway from NHEJ pathway (31). The absence of an MRX complex showed no meiotic DSB-to-JM transition or CO and NCO recombinants, as evidenced by physical analysis of recombination in budding yeast. The presence of unprocessed DSBs induces a checkpoint signal requiring pachytene checkpoint protein 2 (Pch2) that functions with Tel1 and the MRX complex (32). Thus, we theorized that the MRX complex possibly acts together with Pch2 to promote normal meiotic recombination. Herein, we observed diverse recombination 
phenotypes as follows, (i) meiotic recombination and nuclear division progressed normally in the absence of Ku70 as in WT cells; (ii) DSB levels were found reduced at various loci of yeast chromosomes in rad50S ku704 cells; (iii) a large portion of DSBs formed CO and NCO recombinants in rad50S Ku70 during meiosis; and (iv) a subset of DSBs remained unrepaired for $24 \mathrm{~h}$. It has been also reported that NHEJ is suppressed by repression of Nej1 in diploid yeast cells (27). Our results further indicate that $\mathrm{Ku}$ complex is not required for recombination during normal meiosis. Moreover, in the absence of a functional NHEJ and MRX complex, recombination occurred, leading to $\mathrm{CO}$ and $\mathrm{NCO}$ formation. In WT cells, the Ku complex was not essential for $\mathrm{CO}$ and NCO formation, as the MRX complex and Exo1/Dna2-Sgs1 function in forming ssDNA overhangs of DSBs $(33,34)$. When both the MRX complex and Ku complex were defective, Exo1/Dna2-Sgs1 may have functioned to expose ssDNA through their $5^{\prime}$ end resection activity, although this activity was not fully active without the initial strand nicking by the MRX complex (Fig. 4D).

In the present study, we found that the Ku complex is involved in meiotic DSB repair in the absence of MRX activity, but not the presence of the MRX complex. These findings are important for understanding how cells deal with programmed DSBs (or endogenous damage-induced DSBs) during meiosis and how defective DSB end resection affects meiotic recombination in the presence or absence of another repair pathway (35).

\section{MATERIALS AND METHODS}

\section{Yeast strains}

We used the Saccharomyces cerevisiae SK1 strain in this study. Detailed information regarding strains is listed in Supplemental Materials Table S1.

\section{MMS sensitivity test}

Cell were grown in YPD liquid medium (1\% bacto yeast extract, $2 \%$ bacto peptone, and $2 \%$ glucose) for $24 \mathrm{~h}$. Cells were diluted $10^{-1}, 10^{-2}, 10^{-3}, 10^{-4}$, and $10^{-5}$ in distilled water and spotted on YPD plates ( $1 \%$ bacto yeast extract, $2 \%$ bacto peptone, $2 \%$ bacto agar, and $2 \%$ glucose) and YPD plates containing $0.01 \%$ and $0.03 \%$ MMS. The plates were then incubated for 2 days.

\section{Spore viability test}

Diploid cells were grown in SPM ( $1 \%$ potassium acetate, $0.02 \%$ raffinose, and $0.01 \%$ antifoam) for $24 \mathrm{~h}$. Spores were plated onto YPD plates through tetrad dissection and then incubated for 2 days.

\section{Meiotic division}

Cells in SPM were harvested at different time points, fixed in sorbitol solution $(40 \%$ ethanol and $0.1 \mathrm{M}$ sorbitol). Cells were then stained with DAPI $(1 \mu \mathrm{l} / \mathrm{ml})$ and the nuclei were counted $(n=200)$. Nuclei stained with DAPI were observed using fluorescence microscopy (Eclipse Ti-E; Nikon, Tokyo, Japan) and imaged using the Nikon DS-Qi2.

\section{Meiotic time course analysis}

Meiotic time course was performed as described previously (8-11). Cells were streaked onto YPG plates ( $1 \%$ bacto yeast extract, $2 \%$ bacto peptone, $2 \%$ bacto agar, and $3 \%$ glycerol) and incubated overnight. Cells were diluted onto YPD plates and incubated for 2 days. Single colonies were then incubated in YPD liquid media for $18 \mathrm{~h}$. To synchronize cells in G1 phase, a $1 / 500$ dilution of YPD culture was added to SPS $(0.5 \%$ bacto yeast extract, $1 \%$ bacto peptone, $1 \%$ potassium acetate, $0.05 \mathrm{M}$ potassium biphthalate, $0.5 \%$ ammonium sulfate, and $0.17 \%$ yeast nitrogen base without amino acids; $\mathrm{pH}$ 5.5) in a shaking incubator for $18 \mathrm{~h}$. Synchronized cells were then transferred to SPM. Meiotic cells were harvested at different time points and crosslinked with psoralen (Sigma-Aldrich, St. Louis, MO) using ultraviolet light at 360 nm for 15 mins.

\section{Physical analysis of meiotic recombination}

Physical analysis was performed as described previously $(8,9)$. Detailed information regarding the procedures is described in Supplemental Materials.

\section{ACKNOWLEDGEMENTS}

This work was supported by grants to K.P.K. from the National Research Foundation of Korea (NRF) funded by the Korean Ministry of Science, ICT and Future Planning (MSIT; NRF-2017R1A2B2005603; NRF-2018R1A5A1025077) and the Next-Generation BioGreen 21 Program (SSAC; No. PJ01322801), Rural Development Administration, Republic of Korea.

\section{CONFLICTS OF INTEREST}

The authors have no conflicting interests.

\section{REFERENCES}

1. Chung WH (2014) To peep into Pif1 helicase: Multifaceted all the way from genome stability to repair-associated DNA synthesis. J Microbiol 52, 89-98

2. Choi DH, Lee R, Kwon SH and Bae SH (2013) Hrq1 functions independently of Sgs1 to preserve genome integrity in Saccharomyces cerevisiae. J Microbiol 51, 105-112

3. Sung $P$ (2018) Introduction to the Thematic Minireview Series: DNA double-strand break repair and pathway choice. J Biol Chem 293, 10500-10501

4. Haber JE (2018) DNA Repair: The Search for Homology. BioEssays 40, 1700229

5. Fouquin A, Guirouilh-Barbat J, Lopez B, Hall J, 
Amor-Guéret M and Pennaneach V (2017) PARP2 controls double-strand break repair pathway choice by limiting 53BP1 accumulation at DNA damage sites and promoting end-resection. Nucleic Acids Res 45, 1232512339

6. Daley JM, Laan RLV, Suresh A and Wilson TE (2005) DNA Joint dependence of Pol $X$ family polymerase action in nonhomologous end joining. J Biol Chem 280, 29030-29037

7. Cannavo E, Johnson D, Andres SN et al (2018) Regulatory control of DNA end resection by Sae2 phosphorylation. Nat Commun 9, 4016

8. Kim KP, Weiner BM, Zhang L, Jordan A, Dekker J and Kleckner N (2010) Sister cohesion and structural axis components mediate homolog bias of meiotic recombination. Cell 143, 924-937

9. Hong S, Sung Y, Yu M, Lee M, Kleckner N and Kim KP (2013) The logic and mechanism of homologous recombination partner choice. Mol Cell 51, 440-453

10. Hong S and Kim KP (2013) Shu1 promotes homolog bias of meiotic recombination in Saccharomyces cerevisiae. Mol Cells 36, 446-454

11. Yoon SW, Lee MS, Xaver M et al (2016) Meiotic prophase roles of Rec8 in crossover recombination and chromosome structure. Nucleic Acids Res 44, 9296-9314

12. Marcon E and Moens PB (2005) The evolution of meiosis: Recruitment and modification of somatic DNA-repair proteins. Bioessays 27, 795-808

13. Lam I and Keeney S (2015) Mechanism and regulation of meiotic recombination initiation. Cold Spring Harb Perspect Biol 7, a016634-a016634

14. Gobbini E, Cassani C, Villa M, Bonetti D and Longhese MP (2016) Functions and regulation of the MRX complex at DNA double-strand breaks. Microbial Cell 3, 329-337

15. Seeber A, Hegnauer AM, Hustedt $N$ et al (2016) RPA mediates recruitment of MRX to forks and double-strand breaks to hold sister chromatids together. Molecular Cell 64, 951-966

16. Chen H, Donnianni RA, Handa N et al (2015) Sae2 promotes DNA damage resistance by removing the Mre11-Rad50-Xrs2 complex from DNA and attenuating Rad53 signaling. Proc Natl Acad Sci U S A 112, E1880E1887

17. Hefferin ML and Tomkinson AE (2005) Mechanism of DNA double-strand break repair by non-homologous end joining. DNA Repair 4, 639-648

18. Huertas P and Jackson SP (2009) Human CtIP mediates cell cycle control of DNA end resection and double strand break repair. J Biol Chem 284, 9558-9565

19. Huertas P, Cortés-Ledesma F, Sartori AA, Aguilera A and Jackson SP (2008) CDK targets Sae2 to control DNA-end resection and homologous recombination. Nature 455, 689-692

20. Mimitou EP and Symington LS (2011) DNA end resection - unraveling the tail. DNA Repair (Amst) 10, 344-348

21. Kim KP and Mirkin EV (2018) So similar yet so different:
The two ends of a double strand break. Mutat Res 809, 70-80

22. Tsukamoto Y, Kato J and Ikeda H (1996) Hdf1, a yeast Ku-protein homologue, is involved in illegitimate recombination, but not in homologous recombination. Nucleic Acids Res 24, 2067-2072

23. Gu Y, Jin S, Gao Y, Weaver DT and Alt FW (1997) Ku70-deficient embryonic stem cells have increased ionizing radiosensitivity, defective DNA end-binding activity, and inability to support V(D)J recombination. Proc Natl Acad Sci U S A 94, 8076-8081

24. Sorenson KS, Mahaney BL, Lees-Miller SP and Cobb JA (2017) The non-homologous end-joining factor Nej1 inhibits resection mediated by Dna2-Sgs1 nucleasehelicase at DNA double strand breaks. J Biol Chem 292, 14576-14586

25. Pannunzio NR, Watanabe G and Lieber MR (2018) Nonhomologous DNA end-joining for repair of DNA double-strand breaks. J Biol Chem 293, 10512-10523

26. Lieber MR (2010) The mechanism of double-strand DNA break repair by the nonhomologous DNA end-joining pathway. Ann Rev Biochem 79, 181-211

27. Valencia $M$, Bentele $M$, Vaze $M B$ et al (2001) NEJ1 controls non-homologous end joining in Saccharomyces cerevisiae. Nature 414, 666-669

28. Lee MS, Yoon SW and Kim KP (2015) Mitotic cohesin subunit Mcd1 regulates the progression of meiotic recombination in budding yeast. J Microbiol Biotechnol 25, 598-605

29. Cho HR, Kong YJ, Hong SG and Kim KP (2016) Hop2 and Sae3 are required for Dmc1-mediated double-strand break repair via homolog bias during meiosis. Mol Cells 39, 550-556

30. Hong S, Choi EH and Kim KP (2015) Ycs4 is required for efficient double-strand break formation and homologous recombination during meiosis. J Microbiol Biotechnol 25, 1026-1035

31. Yin Y and Smolikove S (2013) Impaired resection of meiotic duble-strand breaks channels repair to nonhomologous end joining in caenorhabditis elegans. Mol Cell Biol 33, 2732-2747

32. Ho HC and Burgess SM (2011) Pch2 Acts through Xrs2 and Tel1/ATM to modulate interhomolog bias and checkpoint function during meiosis. PLoS Genet 7, e1002351

33. Hunter N (2006) Meiotic Recombination. molecular genetics of recombination, Topics in Current Genetics, Springer-Verlag, Heidelberg, 381-442

34. Neale M, Pan J and Keeney S (2005) Endonucleolytic processing of covalent protein-linked DNA double-strand breaks. Nature 436, 1053-1057

35. Devkota S (2018) The road less traveled: strategies to enhance the frequency of homology-directed repair (HDR) for increased efficiency of CRISPR/Cas-mediated transgenesis. BMB Rep 51, 437-443 